\title{
New Similarity Measure between Intuitionistic Fuzzy Multisets based on Tangent Function and its Application in Medical Diagnosis
}

\author{
R. Muthuraj, S. Devi
}

\begin{abstract}
In recent years, Intuitionistic fuzzy set is very useful in decision making problems such as medical diagnosis, pattern recognition, clustering etc., which deals with vagueness and uncertainty. Similarity measure is a tool used to find the closeness of the intuitionistic fuzzy sets by considering the membership, nonmembership and hesitation function. In this paper, we propose an effective similarity measure based on tangent function for intuitionistic fuzzy multi sets in which membership, nonmembership, hesitation function occurs more than once and also we apply this measure in medical diagnosis and pattern recognition.
\end{abstract}

Keywords--- Intuitionistic fuzzy sets(IFS), Intuitionistic Fuzzy Multi sets(IFMS), Similarity measure based on tangent function.

AMS Subject Classification (2010)--- 08A72, $03 E 72$.

\section{INTRODUCTION}

In 1965, L.A.Zadeh[28] introduced the notion of fuzzy subset of a set as a method for representing uncertainty in real physical world. In fuzzy set theory, the membership of an element to a fuzzy set is a single value between 0 and 1 . As a generalization of this, intuitionistic fuzzy subset was defined by K.T.Atanassov in 1986[1,2]. These sets are suited to deal with vagueness or the representation of imperfect knowledge in decision making. In reality, it may not true that the degree of non-membership of an element in an intuitionistic fuzzy set is equal to 1 minus the degree of membership, but there may be some hesitation degree. At first the study of distance measures for IFS was carried out by E.Szmidt and and J.Kacprzyk [22,23,24]. Hung and Yang[6] presented a similarity measure based on Hausdroff distance. Various similarity measures were given by the authors like $\mathrm{Li}$ and Cheng, Liang and Shi, Mitchell[3,4,7,8,9,26,27] which was applied in decision making problem and pattern recognition.

Recently, T.K.Shinoj and Sunil.J.J[20,21] introduced intuitionistic fuzzy multisets from the combination of intuitionistic fuzzy sets and fuzzy multisets which was proposed by Yager[25]. In intuitionistic fuzzy multisets, the membership function and non-membership function are allowed to occur more than once. Some of the distance and similarity measures for IFS were extended to IFMS[5,11,12,13,14,15,16,17,18]. In 2015, K.Mondal and S.Pramanik[10] proposed a similarity measure based on tangent function for IFS. In this paper, we extend this

Revised Manuscript received July 10, 2019.

R. Muthuraj, PG and Research Department of Mathematics, H.H. The Rajah's College, Pudhukottai, Tamilnadu, India.(e-mail: rmr1973@yahoo.co.in)

S. Devi, Department of Mathematics, PSNA College of Engineering and Technology, Dindigul, Tamilnadu, India.

(e-mail: sdevisaran1982@gmail.com) measure for intuitionistic fuzzy multisets and prove its efficiency by comparing the measure with the existing similarity measures for IFMS. Also we present a case study for diagnosing a disease in medical field and also apply this measure in pattern recognition.

\section{PRELIMINARIES}

Definition 2.1 [1]

Let $X$ be a non-empty set. An intuitionistic fuzzy sets A in X is an object having the form

$\mathrm{A}=\left\{\left\langle\mathrm{x}, \mu_{\mathrm{A}}(\mathrm{x}), \nu_{\mathrm{A}}(\mathrm{x})\right\rangle / \mathrm{x} \in \mathrm{X}\right\}$ where the functions $\mu_{\mathrm{A}}$ $: \mathrm{X} \rightarrow[0,1]$ and $v_{\mathrm{A}}: \mathrm{X} \rightarrow[0,1]$ define the degree of membership and non membership of the element $\mathrm{x} \in \mathrm{X}$.

For each IFS A in $\mathrm{X}$, if $\pi_{\mathrm{A}}=1-\mu_{\mathrm{A}}(\mathrm{x})-v_{\mathrm{A}}(\mathrm{x}), \mathrm{x} \in \mathrm{X}$, then $\pi_{\mathrm{A}}(\mathrm{x})$ is called intuitionistic index of the element $\mathrm{x}$ in A. It is a hesitancy degree of $\mathrm{x}$ in $\mathrm{A}$.

\section{Definition 2.2[25]}

Let $\mathrm{X}$ be a nonempty set. A Fuzzy Multiset(FMS) A drawn from $\mathrm{X}$ is characterized by a function, 'count membership' of A denoted by $\mathrm{CM}_{\mathrm{A}}$ such that $\mathrm{CM}_{\mathrm{A}}: \mathrm{X} \rightarrow \mathrm{Q}$ where $\mathrm{Q}$ is the set of all crisp multisets drawn from the unit interval $[0,1]$. Then for any $\mathrm{x} \in \mathrm{X}$, the value $\mathrm{CM}_{\mathrm{A}}(\mathrm{x})$ is a crisp multiset drawn from $[0,1]$. For each $\mathrm{x}$ in $\mathrm{X}$, the membership sequence is defined as the decreasingly ordered sequence of elements in $\mathrm{CM}_{\mathrm{A}}(\mathrm{x})$. It is denoted by $\left(\mu_{A}^{1}(x), \mu_{A}^{2}(x), \ldots \mu_{A}^{P}(x)\right)$ where $\left(\mu_{A}^{1}(x) \geq \mu_{A}^{2}(x) \geq \cdots \geq\right.$ $\left.\mu_{A}^{P}(x)\right)$.

\section{Definition 2.3[20]}

Let $\mathrm{X}$ be a non-empty set . An Intuitionistic Fuzzy Multiset A denoted by IFMS drawn from $X$ is a characterized by two functions: count membership of $\mathrm{A}\left(\mathrm{CM}_{\mathrm{A}}\right)$ and 'count non membership' of $\mathrm{A}\left(\mathrm{CN}_{\mathrm{A}}\right)$ given respectively by $\mathrm{CM}_{\mathrm{A}}: \mathrm{X} \rightarrow \mathrm{Q}$ and $\mathrm{CN}_{\mathrm{A}}: \mathrm{X} \rightarrow \mathrm{Q}$ where $\mathrm{Q}$ is the set of all crisp multisets drawn from the unit interval $[0,1]$ such that for each $\mathrm{x} \in \mathrm{X}$, the membership sequence is defined as a decreasingly ordered sequence of elements in $\mathrm{CM}_{\mathrm{A}}$ (x) which is denoted by $\left(\mu_{A}^{1}(x), \mu_{A}^{2}(x), \ldots \mu_{A}^{P}(x)\right)$ where $\left(\mu_{A}^{1}(x) \geq \mu_{A}^{2}(x) \geq \cdots \geq \mu_{A}^{P}(x)\right)$ and the corresponding non membership sequence will be denoted by $\left(\vartheta_{A}^{1}(x), \vartheta_{A}^{2}(x), \ldots \vartheta_{A}^{P}(x)\right)$ such that $0 \leq \mu_{A}^{i}(x)+\vartheta_{A}^{i}(x) \leq 1$ for every $\mathrm{x} \in \mathrm{X}$ and $\mathrm{i}=1,2, \ldots \mathrm{p}$.

An IFMS A is denoted by 


\section{NEW SIMILARITY MEASURE BETWEEN INTUITIONISTIC FUZZY MULTISETS BASED ON TANGENT FUNCTION AND ITS APPLICATION IN MEDICAL DIAGNOSIS}

$$
\begin{aligned}
& \mathrm{A}=\{\langle\mathrm{x}: \\
& \begin{aligned}
\left.\left(\mu_{A}^{1}(x), \mu_{A}^{2}(x), \ldots \mu_{A}^{P}(x)\right),\left(\vartheta_{A}^{1}(x), \vartheta_{A}^{2}(x), \ldots \vartheta_{A}^{P}(x)\right)\right\rangle: x \\
\quad \in X\} .
\end{aligned}
\end{aligned}
$$

\section{Definition 2.4[21]}

The length of an element $\mathrm{x}$ in an IFMS $\mathrm{A}$ is defined as the cardinality of $\mathrm{CM}_{\mathrm{A}}(\mathrm{x})$ or $\mathrm{CN}_{\mathrm{A}}(\mathrm{x})$ for which $0 \leq \mu_{A}^{j}(x)+$ $\vartheta_{A}^{j}(x) \leq 1$ and it is denoted by $\mathrm{L}(\mathrm{x}: \mathrm{A})$. That is

$$
\mathrm{L}(\mathrm{x}: \mathrm{A})=\left|\mathrm{CM}_{\mathrm{A}}(\mathrm{x})\right|=\left|\mathrm{CN}_{\mathrm{A}}(\mathrm{x})\right|
$$

\section{Definition 2.5[21]}

If $\mathrm{A}$ and $\mathrm{B}$ are IFMS drawn from $\mathrm{X}$ then $\mathrm{L}(\mathrm{x}: \mathrm{A}, \mathrm{B})=$ $\operatorname{Max}\{\mathrm{L}(\mathrm{x}: \mathrm{A}), \mathrm{L}(\mathrm{x}: \mathrm{B})\}$. Alternatively we use $\mathrm{L}(\mathrm{x})$ for $\mathrm{L}(\mathrm{x}$ : $\mathrm{A}, \mathrm{B})$.

\section{Definition 2.6[7]}

A real-valued function $\mathrm{S}: \operatorname{IFS}(\mathrm{X}) \times \operatorname{IFS}(\mathrm{X}) \rightarrow[0,1]$ is called a similarity measure on IFS(X), if it satisfies the axiomatic requirements:

1. $0 \leq \mathrm{S}(\mathrm{A}, \mathrm{B}) \leq 1$

2. $\mathrm{S}(\mathrm{A}, \mathrm{B})=1$ if and only if $\mathrm{A}=\mathrm{B}$

3. $\mathrm{S}(\mathrm{A}, \mathrm{B})=\mathrm{S}(\mathrm{B}, \mathrm{A})$

4. $\mathrm{S}(\mathrm{A}, \mathrm{C}) \leq \mathrm{S}(\mathrm{A}, \mathrm{B})$ and $\mathrm{S}(\mathrm{A}, \mathrm{C}) \leq \mathrm{S}(\mathrm{B}, \mathrm{C})$ if $\mathrm{A} \subseteq \mathrm{B} \subseteq$ C.

\section{Definition 2.7[11]}

The similarity measure based on Hausdroff distance for IFMS is

$\mathrm{S}_{\mathrm{H}}(\mathrm{A}, \mathrm{B})=\frac{1}{\eta} \sum_{j=1}^{\eta}\left[1-\frac{1}{n} \sum_{i=1}^{n} \max \left(\mid \mu_{A}^{j}\left(x_{i}\right)-\right.\right.$ $\mu B j x i, \vartheta A j x i-\vartheta B j x i)$

\section{Definition 2.8[13]}

Cotangent similarity measure for IFMS is IFMS(A,B)

$\frac{1}{\eta} \sum_{j=1}^{\eta}\left[\frac{1}{n} \sum_{i=1}^{n} \cot \left(\frac{\pi+\pi\left(\left|\mu_{A}^{j}\left(x_{i}\right)-\mu_{B}^{j}\left(x_{i}\right)\right| \curlyvee\left|\vartheta_{A}^{j}\left(x_{i}\right)-\vartheta_{B}^{j}\left(x_{i}\right)\right|\right)}{4}\right)\right]$

\section{Definition 2.9[15]}

The similarity measure based on Normalized Hamming distance [15] is

$\operatorname{Sim}(\mathrm{A}, \mathrm{B})=\frac{N_{D}^{*}(A, B)}{N_{D}^{*}\left(A, B^{c}\right)}$

Where $N_{D}^{*}(A, B)=\frac{1}{\eta} \sum_{j=1}^{\eta} \frac{1}{2 n} \sum_{i=1}^{n}\left(\left|\mu_{A}^{j}\left(x_{i}\right)-\mu_{B}^{j}\left(x_{i}\right)\right|+\right.$ vAjxi- vBjxi)

\section{Definition 2.10[19]}

The correlation similarity measure for IFMS[19] is

$$
\rho_{I F M S}=\frac{C_{I F M S}(A, B)}{\sqrt{C_{I F M S}(A, A) * C_{I F M S}(B, B)}}
$$

where $\quad C_{I F M S}(A, B)=\frac{1}{\eta} \sum_{j=1}^{\eta}\left\{\sum_{i=1}^{n}\left(\mu_{A}^{j}\left(x_{i}\right) \mu_{B}^{j}\left(x_{i}\right)+\right.\right.$ vAjxivBjxi),

$C_{I F M S}(A, A)=\frac{1}{\eta} \sum_{j=1}^{\eta}\left\{\sum_{i=1}^{n}\left(\mu_{A}^{j}\left(x_{i}\right) \mu_{A}^{j}\left(x_{i}\right)+\right.\right.$

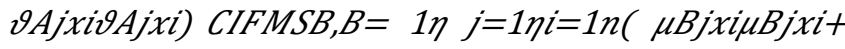

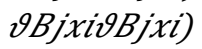

\section{Definition 2.11[16]}

Cosine similarity measure for IFMS[16] is
$\mathrm{C}_{\mathrm{IFM}}(\mathrm{A}, \mathrm{B})=$
$\frac{1}{\eta} \sum_{j=1}^{\eta}\left\{\frac{1}{n} \sum_{i=1}^{n} \frac{\mu_{A}^{j}\left(x_{i}\right) \mu_{B}^{j}\left(x_{i}\right)+\vartheta_{A}^{j}\left(x_{i}\right) \vartheta_{B}^{j}\left(x_{i}\right)}{\sqrt{\mu_{A}^{j}\left(x_{i}\right)^{2}+\vartheta_{A}^{j}\left(x_{i}\right)^{2}} \sqrt{\mu_{B}^{j}\left(x_{i}\right)^{2}+\vartheta_{B}^{j}\left(x_{i}\right)^{2}}}\right\}$

\section{Definition 2.12[10]}

The tangent similarity measure between two IFS P and Q can be presented as

$\mathrm{T}_{\mathrm{IFS}}(\mathrm{P}, \mathrm{Q})=$

$\frac{1}{n} \sum_{i=1}^{n}[1$

$\left.-\tan \left(\frac{\pi\left(\left|\mu_{P}\left(x_{i}\right)-\mu_{Q}\left(x_{i}\right)\right|+\left|v_{P}\left(x_{i}\right)-v_{Q}\left(x_{i}\right)\right|+\left|\xi_{P}\left(x_{i}\right)-\xi_{Q}\left(x_{i}\right)\right|\right)}{12}\right)\right]$

\section{PROPOSED SIMILARITY MEASURE FOR INTUITIONISTIC FUZZY MULTISETS}

\section{Definition 3.1}

The new similarity measure for IFMS based on tangent function with two parameters membership and nonmembership function is

$\mathrm{T}_{\mathrm{IFMS}}(\mathrm{A}, \mathrm{B})=$

$\frac{1}{\eta} \sum_{j=1}^{\eta}[1$

$\left.-\frac{1}{n} \sum_{i=1}^{n} \tan \left(\frac{\pi\left(\left|\mu_{A}^{j}\left(x_{i}\right)-\mu_{B}^{j}\left(x_{i}\right)\right|+\left|\vartheta_{A}^{j}\left(x_{i}\right)-\vartheta_{B}^{j}\left(x_{i}\right)\right|\right)}{12}\right)\right]$

The new similarity measure for IFMS based on tangent function with three parameters membership, nonmembership and hesitation function is

$\mathrm{T}_{\mathrm{IFMS}}(\mathrm{A}, \mathrm{B})=$

$\frac{1}{\eta} \sum_{j=1}^{\eta}[1$

$\left.-\frac{1}{n} \sum_{i=1}^{n} \tan \left(\frac{\pi\left(\left|\mu_{A}^{j}\left(x_{i}\right)-\mu_{B}^{j}\left(x_{i}\right)\right|+\left|\vartheta_{A}^{j}\left(x_{i}\right)-\vartheta_{B}^{j}\left(x_{i}\right)\right|+\left|\Pi_{A}^{j}\left(x_{i}\right)-\Pi_{B}^{j}\left(x_{i}\right)\right|\right)}{12}\right)\right]$

\section{Preposition 3.2}

The defined new similarity measure $\mathrm{T}_{\mathrm{IMFS}}(\mathrm{A}, \mathrm{B})$ between IFMS $A$ and $B$ satisfies the following properties

P1. $0 \leq \mathrm{T}_{\mathrm{IFMS}}(\mathrm{A}, \mathrm{B}) \leq 1$

P2. $\mathrm{T}_{\mathrm{IFMS}}(\mathrm{A}, \mathrm{B})=1$ if and only if $\mathrm{A}=\mathrm{B}$

P3. $\mathrm{T}_{\mathrm{IFMS}}(\mathrm{A}, \mathrm{B})=\mathrm{T}_{\mathrm{IFMS}}(\mathrm{B}, \mathrm{A})$

P4. If $A \subseteq B \subseteq C$, then $T_{I F M S}(A, C) \leq T_{I F M S}(A, B)$ and $\mathrm{T}_{\mathrm{IFMS}}(\mathrm{A}, \mathrm{C}) \leq \mathrm{T}_{\mathrm{IFMS}}(\mathrm{B}, \mathrm{C})$.

Proof:

P1. $0 \leq \mathrm{T}_{\text {IFMS }}(\mathrm{A}, \mathrm{B}) \leq 1$

Since the values of membership, non-membership and hesitation functions of the intuitionistic fuzzy multiset are lying in the interval $[0,1]$, the similarity measure based on tangent function $\mathrm{T}_{\mathrm{IFMS}}(\mathrm{A}, \mathrm{B})$ is lying between 0 and 1 .

P2. $T_{\text {IFMS }}(A, B)=1$ if and only if $A=B$

i)If the two IFMS $\mathrm{A}$ and $\mathrm{B}$ are equal, then $\mu_{A}^{j}\left(x_{i}\right)=$ $\mu_{B}^{j}\left(x_{i}\right), \vartheta_{A}^{j}\left(x_{i}\right)=\vartheta_{B}^{j}\left(x_{i}\right)$ and $\Pi_{A}^{j}\left(x_{i}\right)=\Pi_{B}^{j}\left(x_{i}\right) \forall \mathrm{j}$, which implies that $\left|\mu_{A}^{j}\left(x_{i}\right)-\mu_{B}^{j}\left(x_{i}\right)\right|=0,\left|\vartheta_{A}^{j}\left(x_{i}\right)-\vartheta_{B}^{j}\left(x_{i}\right)\right|=$ $0,\left|\Pi_{A}^{j}\left(x_{i}\right)-\Pi_{B}^{j}\right|=0$ 
Hence $\mathrm{T}_{\mathrm{IFMS}}(\mathrm{A}, \mathrm{B})=1$.

ii) Let $\mathrm{T}_{\mathrm{IFMS}}(\mathrm{A}, \mathrm{B})=1$.Then $\left|\mu_{A}^{j}\left(x_{i}\right)-\mu_{B}^{j}\left(x_{i}\right)\right|=$ $0,\left|\vartheta_{A}^{j}\left(x_{i}\right)-\vartheta_{B}^{j}\left(x_{i}\right)\right|=0,\left|\Pi_{A}^{j}\left(x_{i}\right)-\Pi_{B}^{j}\right|=0$ This implies that $\quad \mu_{A}^{j}\left(x_{i}\right)=\mu_{B}^{j}\left(x_{i}\right), \vartheta_{A}^{j}\left(x_{i}\right)=\vartheta_{B}^{j}\left(x_{i}\right)$ and $\Pi_{A}^{j}\left(x_{i}\right)=$ $\Pi_{B}^{j}\left(x_{i}\right)$ for all $\mathrm{j}$. Thus $\mathrm{A}=\mathrm{B}$.

P3. $\mathbf{T}_{\text {IFMS }}(\mathbf{A}, B)=\mathbf{T}_{\text {IFMS }}(B, A)$

$\mathrm{T}_{\text {IFMS }}(\mathrm{A}, \mathrm{B})=$

$\frac{1}{\eta} \sum_{j=1}^{\eta}[1$

$\left.-\frac{1}{n} \sum_{i=1}^{n} \tan \left(\frac{\pi\left(\left|\mu_{A}^{j}\left(x_{i}\right)-\mu_{B}^{j}\left(x_{i}\right)\right|+\left|\vartheta_{A}^{j}\left(x_{i}\right)-\vartheta_{B}^{j}\left(x_{i}\right)\right|+\left|\Pi_{A}^{j}\left(x_{i}\right)-\Pi_{B}^{j}\left(x_{i}\right)\right|\right.}{12}\right)\right]$

$=$

$\frac{1}{\eta} \sum_{j=1}^{\eta}[1-$

1n $i=1 n \tan \pi \quad(\mu B j x i-\quad \mu A j x i+\quad \vartheta B j x i-$ $\vartheta \operatorname{Ajxi}+\Pi B j(x i)-\Pi A j(x i) 12$

$=\mathrm{T}_{\text {IFMS }}(\mathrm{A}, \mathrm{B})$

P4. If $A \subseteq B \subseteq C$, then $T_{\text {IFMS }}(A, C) \leq T_{\text {IFMS }}(A, B)$ and $\mathbf{T}_{\text {IFMS }}(A, C) \leq \mathbf{T}_{\text {IFMS }}(B, C)$

If $\mathrm{A} \subseteq \mathrm{B} \subseteq \mathrm{C}$, then $\mu_{A}^{j}\left(x_{i}\right) \leq \mu_{B}^{j}\left(x_{i}\right) \leq \mu_{C}^{j}\left(x_{i}\right), \vartheta_{A}^{j}\left(x_{i}\right) \geq$ $\vartheta_{B}^{j}\left(x_{i}\right) \geq \vartheta_{C}^{j}\left(x_{i}\right)$ and $\Pi_{A}^{j}\left(x_{i}\right) \geq \Pi_{B}^{j}\left(x_{i}\right) \geq \Pi_{C}^{j}\left(x_{i}\right)$. We have

$\left|\mu_{A}^{j}\left(x_{i}\right)-\mu_{B}^{j}\left(x_{i}\right)\right| \leq\left|\mu_{A}^{j}\left(x_{i}\right)-\mu_{C}^{j}\left(x_{i}\right)\right|, \mid \mu_{B}^{j}\left(x_{i}\right)-$

$\mu C j(x i) \leq \mu A j x i-\mu C j(x i)$

$\left|\vartheta_{A}^{j}\left(x_{i}\right)-\vartheta_{B}^{j}\left(x_{i}\right)\right| \leq\left|\vartheta_{A}^{j}\left(x_{i}\right)-\vartheta_{C}^{j}\left(x_{i}\right)\right|, \mid \vartheta_{B}^{j}\left(x_{i}\right)-$

$\vartheta C_{j}(x i) \leq \vartheta A j x i-\vartheta C j(x i)$

and $\left|\Pi_{A}^{j}\left(x_{i}\right)-\Pi_{B}^{j}\left(x_{i}\right)\right| \leq\left|\Pi_{A}^{j}\left(x_{i}\right)-\Pi_{C}^{j}\left(x_{i}\right)\right|, \mid \Pi_{B}^{j}\left(x_{i}\right)-$ $\Pi C j(x i) \leq \Pi A j x i-\Pi C j(x i)$

Hence $\mathrm{T}_{\text {IFMS }}(\mathrm{A}, \mathrm{C}) \leq \mathrm{T}_{\mathrm{IFMS}}(\mathrm{A}, \mathrm{B})$ and $\mathrm{T}_{\mathrm{IFMS}}(\mathrm{A}, \mathrm{C}) \leq$ $\mathrm{T}_{\mathrm{IFMS}}(\mathrm{B}, \mathrm{C})$

\section{SIGNIFICANCE OF THE PROPOSED SIMILARITY MEASURE}

\section{Example 4.1}

Let $X=\left\{A_{1}, A_{2}, A_{3}, A_{4}, \ldots . A_{n}\right\}$ with the IFMS's $A=\left\{A_{1}, A_{2}\right\}$ , $B=\left\{A_{1}, A_{3}\right\}$ and $C=\left\{A_{1}, A_{4}\right\}$ defined as $A=\left\{\left\langle A_{1}\right.\right.$ : $\left.(0.2,0.2)\rangle,\left\langle\mathrm{A}_{2}:(0.4,0.4)\right\rangle\right\}, \mathrm{B}=\left\{\left\langle\mathrm{A}_{1}:(0.2,0.2)\right\rangle\right.$, $\left.\left\langle\mathrm{A}_{3}:(0.3,0.3)\right\rangle\right\}$ and $\mathrm{C}=\left\{\left\langle\mathrm{A}_{1}:(0.2,0.2)\right\rangle,\left\langle\mathrm{A}_{4}\right.\right.$ : $(0.301,0.299)\rangle\}$

Here the cardinality $\eta=2$ as $\left|\mathrm{CM}_{\mathrm{A}}\right|=\left|\mathrm{CN}_{\mathrm{A}}\right|=2$, $\mid$ $\mathrm{CM}_{\mathrm{B}}|=| \mathrm{CN}_{\mathrm{B}} \mid=2$, $\left.\mid \mathrm{CMc}\right) \mid=$

$|\mathrm{CNc}|=2$.

Then by the correlation measure for IFMS, $\rho_{I F M S}(\mathrm{~A}, \mathrm{~B})=$ $0.9923, \rho_{I F M S}(\mathrm{~B}, \mathrm{C})=1$. This implies that $\mathrm{B}=\mathrm{C}$ which cannot be true. By Cosine similarity measure for IFMS, $\mathrm{C}_{\mathrm{IFM}}(\mathrm{A}, \mathrm{B})=1, \mathrm{C}_{\mathrm{IFM}}(\mathrm{B}, \mathrm{C})=1$. This implies that the IFMS $\mathrm{A}, \mathrm{B}$ and $\mathrm{C}$ are equal which is not acceptable.

But in our proposed similarity measure, $\mathrm{T}_{\mathrm{IFMS}}(\mathrm{A}, \mathrm{B})=$ 0.9737, $T_{\text {IFMS }}(B, C)=\mathbf{0 . 9 9 9 4}$ in which the similarities of the IFMS A,B and $\mathrm{C}$ can be differentiated and we get the result that $\mathrm{B}$ is more similar to $\mathrm{C}$.

\section{Example 4.2}

Let $X=\left\{A_{1}, A_{2}, A_{3}, A_{4}, \ldots . A_{n}\right\}$ with the IFMS's $A=\left\{A_{1}, A_{2}\right\}$ , $B=\left\{A_{1}, A_{3}\right\}$ and $C=\left\{A_{1}, A_{4}\right\}$ defined as $A=\left\{\left\langle A_{1}\right.\right.$ : $\left.(0.2,0.3)\rangle,\left\langle A_{2}:(0.3,0.3)\right\rangle\right\}, B=\left\{\left\langle A_{1}:(0.2,0.3)\right\rangle\right.$,
$\left.\left\langle\mathrm{A}_{3}:(0.1,0.2)\right\rangle\right\}$ and $\mathrm{C}=\left\{\left\langle\mathrm{A}_{1}:(0.2,0.3)\right\rangle,\left\langle\mathrm{A}_{4}:(0.1,0.1)\right\rangle\right.$ \} .

Here the cardinality $\eta=2$ as $\left|\mathrm{CM}_{\mathrm{A}}\right|=\left|\mathrm{CN}_{\mathrm{A}}\right|=2$, । $\mathrm{CM}_{\mathrm{B}}|=| \mathrm{CN}_{\mathrm{B}} \mid=2$, $\left.\mid \mathrm{CMc}\right) \mid=$

$|\mathrm{CNc}|=2$.

\begin{tabular}{|c|c|c|}
\hline Similarity measures & $(\mathbf{A}, \mathbf{B})$ & $(A, C)$ \\
\hline $\begin{array}{l}\text { Cotangent similarity } \\
\text { measure for IFMS }\end{array}$ & 0.8633 & 0.8633 \\
\hline $\begin{array}{l}\text { Similarity measure based } \\
\text { on Hausdroff distance for } \\
\text { IFMS }\end{array}$ & 0.9 & 0.9 \\
\hline $\begin{array}{l}\text { Similarity measure based } \\
\text { on Hamming distance for } \\
\text { IFMS }\end{array}$ & 0.6 & 0.66 \\
\hline $\begin{array}{l}\text { Proposed Similarity } \\
\text { measure based on tangent } \\
\text { function for IFMS }\end{array}$ & 0.9606 & 0.9474 \\
\hline
\end{tabular}

From the table, we infer that we cannot differentiate the similarity of $(A, B)$ and $(A, C)$ by using the cotangent similarity measure, Similarity measure based on Hausdroff distance and Similarity measure based on Hamming distance. But by the proposed similarity measure based on tangent function, we get the result that $\mathrm{A}$ is more similar to B.

\section{MEDICAL DIAGNOSIS USING SIMILARITY MEASURE BASED ON TANGENT FUNCTION FOR IFMS}

Most of the human reasoning involves the use of variables whose values are uncertain. These uncertainties are denoted by membership values in fuzzy sets. But in some situations like medical diagnosis, the terms of membership function is not adequate.

Hence intuitionistic fuzzy set theory can be used because it consisting of both membership and non-membership functions of an elemlent in a set. Some times each element has different membership values and non-membership values. In such cases, intuitionistic fuzzy multisets can be applied. Here we take the case study of childhood diseases with common symptoms. Nowadays, the process of classifying different set of symptoms under a single name of disease is more difficult. The proposed similarity measure among Children Vs Symptoms and Symptoms Vs Diseases give proper medical diagnosis.

Let $\mathrm{C}=\left\{\mathrm{C}_{1}, \mathrm{C}_{2}, \mathrm{C}_{3}\right\}$ be a set of children.

$\mathrm{D}=\{$ Viral fever, Throat problem, Chickenpox, Skin problem, Mumps $\}$ be the set of diseases and $S=\{$ Temperature, Headache, Throat pain, Muscle ache, Spots $\}$ be the set of symptoms.

Table 1 contains some diseases and their symptoms in intuitionistic fuzzy set values. 


\section{NEW SIMILARITY MEASURE BETWEEN INTUITIONISTIC FUZZY MULTISETS BASED ON TANGENT FUNCTION AND ITS APPLICATION IN MEDICAL DIAGNOSIS}

We observed that, the diseases have the same symptoms but in different proportions. Each symptom is described by three numbers: membership $\mu$, non-membership $v$ and hesitation margin $\pi$.

Table 1: Symptoms Vs Diseases

\begin{tabular}{|l|l|l|l|l|l|}
\hline & $\begin{array}{l}\text { Viral } \\
\text { fever }\end{array}$ & $\begin{array}{l}\text { Throat } \\
\text { proble } \\
\mathrm{m}\end{array}$ & $\begin{array}{l}\text { Chicke } \\
\text { npox }\end{array}$ & $\begin{array}{l}\text { Skin } \\
\text { proble } \\
\mathrm{m}\end{array}$ & Mumps \\
\hline $\begin{array}{l}\text { Temper } \\
\text { ature }\end{array}$ & $(0.8,0.2$ & $(0.2,0.5$ & $(0.9,0.1$ & $(0.1,0.6$ & $(0.6,0.2$ \\
\hline Headac & $(0.7,0.1$ & $, 0.3)$ & $, 0)$ & $, 0.3)$ & $, 0.2)$ \\
he & $, 0.2)$ & $, 0)$ & $, 0.1)$ & $, 0.2)$ & $, 0.2)$ \\
\hline Throat & $(0.2,0.6$ & $(0.8,0.1$ & $(0.3,0.5$ & $(0.3,0.7$ & $(0.8,0.1$ \\
pain & $, 0.2)$ & $, 0.1)$ & $, 0.2)$ & $, 0)$ & $, 0.1)$ \\
\hline Muscle & $(0.5,0.3$ & $(0.4,0.3$ & $(0.5,0.4$ & $(0.3,0.6$ & $(0.7,0.1$ \\
ache & $, 0.2)$ & $, 0.3)$ & $, 0.1)$ & $, 0.1)$ & $, 0.2)$ \\
\hline Spots & $(0.2,0.5$ & $(0.1,0.7$ & $(0.8,0.1$ & $(0.6,0.1$ & $(0.5,0.1$ \\
& $, 0.3)$ & $, 0.2)$ & $, 0.1)$ & $, 0.3)$ & $, 0.4)$ \\
\hline
\end{tabular}

The objective is to make a proper diagnosis for each children. Let the samples be taken at three different timings in a day(morning, noon, night). After the samples obtained, we get a supposed medical analysis of the Children as shown in Table 2.

Table 2: Children Vs Symptoms

\begin{tabular}{|l|l|l|l|l|l|}
\hline & $\begin{array}{l}\text { Tempera } \\
\text { ture }\end{array}$ & $\begin{array}{l}\text { Headach } \\
\mathrm{e}\end{array}$ & $\begin{array}{l}\text { Throat } \\
\text { pain }\end{array}$ & $\begin{array}{l}\text { Muscle } \\
\text { ache }\end{array}$ & Spots \\
\hline \multirow{4}{*}{$\mathrm{C}$} & $(0.2,0.4$, & $(0.2,0.6$, & $(0.8,0.1$, & $(0.5,0.2$, & $(0.2,0.6$, \\
& $0.4)$ & $0.2)$ & $0.1)$ & $0.3)$ & $0.2)$ \\
& $(0.6,0.2$, & $(0.4,0.3$, & $(0.7,0.1$, & $(0.6,0.3$, & $(0,0.7,0$. \\
& $0.2)$ & $0.3)$ & $0.2)$ & $0.1)$ & $3)$ \\
& $(0.3,0.5$, & $(0.5,0.2$, & $(0.9,0.1$, & $(0.7,0.2$, & $(0.1,0.8$, \\
& $0.2)$ & $0.3)$ & $0)$ & $0.1)$ & $0.1)$ \\
\hline & $(0.8,0.1$, & $(0.7,0.2$, & $(0.1,0.7$, & $(0.6,0.2$, & $(0.7,0.1$, \\
$\mathrm{C}$ & $0.1)$ & $0.1)$ & $0.2)$ & $0.2)$ & $0.2)$ \\
2 & $(0.9,0.1$, & $(0.6,0.1$, & $(0.2,0.7$, & $(0.8,0.1$, & $(0.8,0.1$, \\
& $0)$ & $0.3)$ & $0.1)$ & $0.1)$ & $0.1)$ \\
& $(0.6,0.2$, & $(0.5,0.2$, & $(0,0.6,0$. & $(0.7,0.3$, & $(0.6,0.1$, \\
& $0.2)$ & $0.3)$ & $4)$ & $0)$ & $0.3)$ \\
\hline & $(0.7,0.2$, & $(0.6,0.2$, & $(0.3,0.6$, & $(0.5,0.1$, & $(0.2,0.8$, \\
& $0.1)$ & $0.2)$ & $0.1)$ & $0.4)$ & $0)$ \\
$\mathrm{C}$ & $(0.6,0.3$, & $(0.4,0.4$, & $(0.4,0.3$, & $(0.4,0.1$, & $(0.4,0.5$, \\
3 & $0.1)$ & $0.2)$ & $0.3)$ & $0.5)$ & $0.1)$ \\
& $(0.8,0.1$, & $(0.5,0.2$, & $((0.5,0.2$, & $(0.6,0.1$, & $(0.1,0.7$, \\
& $0.1)$ & $0.3)$ & $0.3)$ & $0.3)$ & $0.2)$ \\
\hline
\end{tabular}

Table 3: Proposed Similarity Measure Based On Tangent Function between Children \& Diseases

\begin{tabular}{|l|l|l|l|l|l|}
\hline & Viral fever & Throat problem & Chickenpox & Skin problem & Mumps \\
\hline $\mathrm{C}_{1}$ & 0.8179 & $\mathbf{0 . 9 1 2 0}$ & 0.7586 & 0.7744 & 0.8681 \\
\hline $\mathrm{C}_{2}$ & 0.8833 & 0.7036 & $\mathbf{0 . 9 1 2 3}$ & 0.7956 & 0.8529 \\
\hline $\mathrm{C}_{3}$ & $\mathbf{0 . 8 9 1 1}$ & 0.8188 & 0.8289 & 0.7634 & 0.8468 \\
\hline
\end{tabular}

The proper diagnosis for the children is derived from the highest value of the similarity measure in table 3 . We get the result that Child $\mathrm{C}_{1}$ suffers from Throat problem, Child $\mathrm{C}_{2}$ suffers from Chickenpox, Child $\mathrm{C}_{3}$ suffers from Viral fever.

\section{PATTERN RECOGNITION BY USING THE PROPOSED SIMILARITY MEASURE \& RESULTS}

In this section, some testing patterns can be classified by employing the proposed similarity measure for IFMS.

\section{Example 6.1}

Let Pattern I, Pattern II be the two IFMS's defined as Pattern $I=\left\{\left\langle A_{1}:(0.4,0.3),(0.5,0.4)\right\rangle,\left\langle A_{2}:(0.6,0.4)\right.\right.$, $(0.5,0.5)\rangle,\left\langle\mathrm{A}_{3}:(0.5,0.2),(0.3,0.4)\right\rangle,\langle$

$\left.\left.\mathrm{A}_{4}:(0.3,0.1),(0.3,0.1)\right\rangle,\left\langle\mathrm{A}_{5}:(0.3,0.2),(0.2,0.2)\right\rangle\right\}$,

Pattern II $=\left\{\left\langle\mathrm{A}_{2}:(0.6,0.4),(0.5,0.5)\right\rangle,\left\langle\mathrm{A}_{5}:(0.3,0.2)\right.\right.$, $(0.2,0.2)\rangle,\left\langle\mathrm{A}_{7}:(0.5,0.3),(0.6,0.2)\right\rangle,\langle$

$\left.\left.\mathrm{A}_{8}:(0.7,0.1),(0.1,0.1)\right\rangle,\left\langle\mathrm{A}_{9}:(0.8,0.2),(0.7,0.1)\right\rangle\right\}$

The testing Pattern III is the IFMS defined as

Pattern III $=\left\{\left\langle\mathrm{A}_{6}:(0.6,0.2),(0.3,0.4)\right\rangle,\left\langle\mathrm{A}_{7}:(0.5,0.3)\right.\right.$, $(0.6,0.2)\rangle,\left\langle A_{8}:(0.7,0.1),(0.1,0.1)\right\rangle,\langle$

$\left.\left.\mathrm{A}_{9}:(0.8,0.2),(0.7,0.1)\right\rangle,\left\langle\mathrm{A}_{10}:(0,0.7),(0.1,0.6)\right\rangle\right\}$

Here the cardinality $\eta=5$ as $\mid \mathrm{CM}($ Pattern $\mathrm{I}) \mid=1$ $\mathrm{CN}($ Pattern I) $\mid=5$, | CM(Pattern II $)|=| \mathrm{CN}($ Pattern II $) \mid$ $=5, \mid \mathrm{CM}($ Pattern III $)|=| \mathrm{CN}($ Pattern III $) \mid=5$

The proposed similarity measure between Pattern I and Pattern III is $\mathbf{0 . 8 8 9 3 .}$

The proposed similarity measure between Pattern II and Pattern III is 0.8561 .

Hence the testing Pattern III belongs to Pattern I.

\section{Example 6.2}

Let $X=\left\{A_{1}, A_{2}, \ldots . . A_{n}\right\}$ be a non empty set. Let the patterns $A=\left\{A_{1}, A_{2}\right\}, B=\left\{A_{4}, A_{5}\right\}, C=\left\{A_{1}, A_{8}\right\}, \quad D=$ $\left\{A_{3}, A_{6}\right\}, E=\left\{A_{1}, A_{5}\right\}$ be the IFMSs defined as

$A=\left\{\left\langle A_{1}:(0.1,0.2)\right\rangle,\left\langle A_{2}:(0.1,0.3)\right\rangle\right\}$

$B=\left\{\left\langle A_{4}:(0.3,0.3)\right\rangle,\left\langle A_{5}:(0.2,0.3)\right\rangle\right\}$

$\mathrm{C}=\left\{\left\langle\mathrm{A}_{1}:(0.1,0.2)\right\rangle,\left\langle\mathrm{A}_{8}:(0,0.2)\right\rangle\right\}$

$\mathrm{D}=\left\{\left\langle\mathrm{A}_{3}:(0.3,0.2)\right\rangle,\left\langle\mathrm{A}_{6}:(0.1,0.1)\right\rangle\right\}$

$E=\left\{\left\langle A_{1}:(0.1,0.2)\right\rangle,\left\langle A_{5}:(0.2,0.3)\right\rangle\right\}$

And the testing pattern $Y=\left\{\left\langle A_{6}:(0.1,0.1)\right\rangle,\left\langle A_{8}:(0,0.2)\right\rangle\right.$ \}

Here the cardinality $\eta=2$.

By the proposed similarity measure, $\mathrm{T}_{\mathrm{IFMS}}(\mathrm{A}, \mathrm{Y})=0.9607$,

$\mathrm{T}_{\mathrm{IFMS}}(\mathrm{B}, \mathrm{Y})=0.9081, \mathrm{~T}_{\mathrm{IFMS}}(\mathrm{C}, \mathrm{Y})=\mathbf{0 . 9 8 6 9}, \mathrm{T}_{\mathrm{IFMS}}(\mathrm{D}, \mathrm{Y})=$ $0.9344, \mathrm{~T}_{\mathrm{IFMS}}(\mathrm{E}, \mathrm{Y})=0.9476$.

Hence the testing Pattern Y belongs to Pattern C.

\section{Example 6.3}

Let $X=\left\{A_{1}, A_{2}, \ldots . . A_{n}\right\}$ be a non empty set. Let the patterns $A=\left\{A_{1}, A_{2}\right\}, B=\left\{A_{3}, A_{4}\right\}, C=\left\{A_{5}, A_{6}\right\}$ be the IFMS's defined as

$A=\left\{\left\langle A_{1}:(0.4,0.3,0.3),(0.5,0.3,0.2)\right\rangle,\left\langle A_{2}:(0.6,0.2,0.2)\right.\right.$, $(0.4,0.2,0.4)\rangle\}$

$\mathrm{B}=\left\{\left\langle\mathrm{A}_{3}:(0.7,0.1,0.2),(0.6,0.3,0.1)\right\rangle,\left\langle\mathrm{A}_{4}:(0.5,0.2,0.3)\right.\right.$, $(0.6,0.2,0.2)\rangle\}$

$\mathrm{C}=\left\{\left\langle\mathrm{A}_{5}:(0.3,0.6,0.1),(0.2,0.4,0.4)\right\rangle,\left\langle\mathrm{A}_{6}:(0.7,0.2,0.1)\right.\right.$, $(0.5,0.4,0.1)\rangle\}$

The testing pattern $\mathrm{D}$ is $\left\langle\mathrm{A}_{1}:(0.4,0.3,0.3),(0.5,0.3,0.2)\right\rangle,\langle$ $\left.\left.\mathrm{A}_{6}:(0.7,0.2,0.1),(0.5,0.4,0.1)\right\rangle\right\}$

Here the cardinality $\eta=2$ and number of elements $\mathrm{n}=2$.

By the proposed similarity measure, $\mathrm{T}_{\mathrm{IFMS}}(\mathrm{A}, \mathrm{D}) \mathbf{= 0 . 9 4 7 3}$,

$\mathrm{T}_{\text {IFMS }}(\mathrm{B}, \mathrm{D})=0.8948, \mathrm{~T}_{\text {IFMS }}(\mathrm{C}, \mathrm{D})=0.9208$

Hence the testing pattern $\mathrm{D}$ belongs to pattern A. 


\section{REFERENCES}

1. Atanassov.K.T.,Intuitionistic fuzzy sets, Fuzzy Sets and Systems, Vol.20, No.1(1986) 87-96.

2. Atanassov.K.T., More on Intuitionistic fuzzy sets, Fuzzy Sets and System, 33 (1989), 37-46.

3. Dey.S.K, Biswas.R, Roy.A.R., An application of intuitionistic fuzzy sets in medical diagnosis, Fuzzy Sets and System, 117(2) (2001), 209-213.

4. Dey.S.K., Biswas.R, Roy.A.R., Some operations on intuitionistic fuzzy sets, Fuzzy Sets and System, 114 (2000), 477-484.

5. Ejegwa.P.A., On Intuitionistic Fuzzy Multisets Theory and Its Application in Diagnostic Medicine, MAYFEB Journal of Mathematics, Vol.4 (2016), 13-22.

6. Hung.W.L. and Yang.M.S., Similarity measures of intuitionistic fuzzy sets based on Hausdroff distance, Pattern Recognition Letters, vol.25, No.14(2004), 16031611.

7. Li.D, Cheng.C., New Similarity measures on Intuitionistic fuzzy sets and application to pattern recognition, Pattern Recognition Letters, 23 (2002), 221 225.

8. Liang.Z, Shi.P., Similarity measures on Intuitionistic fuzzy sets, Pattern Recognition Letters, 24 (2003), 2687 2693.

9. Mitchell, H.B., On the Dengfeng-Chuntian similarity measure and its application to pattern recognition, Pattern Recognition letters, vol.24, No.16(2003), 3101-3104.

10. Mondal.K, Paramanik.S., Intuitionistic Fuzzy Similarity Measure Based on Tangent Functrion and Its Application To Multi-Attribute Decision Making, Global Journal of Academic Research, vol.2, No.2 (2015), 464-471.

11. Rajarajeswari.P., and Uma.N., Hausdroff Similarity Measures for Intuitionistic Fuzzy Multi Sets and Its Application in Medical Diagnosis, International Journal of Mathematical Archive, vol.4(9) (2013), 106-111.

12. Rajarajeswari.P and Uma.N., On Distance and Similarity Measures of Intuitionistic Fuzzy Multi Set, IOSR Journal of Mathematics(IOSR-JM), vol.5, Issue 4 (2013), 19-23.

13. Rajarajeswari.P and Uma.N., Intuitionistic Fuzzy Multi Similarity Measure based on Cotangent function, International Journal of Engineering Research and Technology, vol.2, No.11(2013), 1323-1329.

14. Rajarajeswari.P and Uma.N., A Study of normalized geometric and normalized Hamming distance measures in intuitionistic fuzzy multisets, Int. Journal of Science and Research, Vol.2, N0.11 (2013), 76-80.

15. Rajarajeswari.P and Uma.N, Normalized Hamming Similarity Measure for Intuitionistic Fuzzy Multisets and Its Application in Medical diagnosis, Int.Journal of Mathematics Trends and Technology, Vol.5, No.3 (2014), 219-225.

16. Rajarajeswari.P and Uma.N.,Intuitionistic fuzzy multi similarity measure based on cosine function, International Journal of Research in Information Technology, vol.2, No.3(2014), 581-589.

17. Rajarajeswari.P and Uma.N., The Zhang and Fu's Similarity measure on Intuitionistic fuzzy multi sets, International Journal of Innovative Research in Science, Engineering and Technology, vol.3, No.5(2014), 1230912317.

18. Rajarajeswari.P and Uma.N., A New similarity measure of Intuitionistic fuzzy multi sets in Medical diagonosis application, International Journal of Pure and Applied Mathematics, vol.119, No.17(2018), 859-872.

19. Rajarajeswari.P and Uma.N, Correlation measure for Intuitionistic fuzzy multi sets, International Journal of Research in Engineering and Technology, vol.3, issue 1(2014), 611-617.
20. Shinoj, T.K and Sunil.J.J., Intuitionistic Fuzzy Multisets, International Journal of Engineering Science and Innovative Technology, Vol.2, No.6 (2013), 1-24.

21. Shinoj T.K. and Sunil J.J, Intuitionistic Fuzzy Multisets And Its Application in Medical Diagnosis, International Journal of Mathematical and Computational Sciences, Vol.6, No.1 (2012), 121-124.

22. Szmidt E., Kacprzyk J., Distances between intuitionistic fuzzy Sets, Fuzzy Sets and Systems, 114 (2000), 505518 .

23. Szmidt E., Kacprzyk J., On measuring distances between intuitionistic fuzzy sets, Notes on IFS, vol.3 (1997), 1-13.

24. Szmidt E., Kacprzyk J., Distances between intuitionistic fuzzy sets: Straightforward Approaches may not work. $3^{\text {rd }}$ Int. IEEE Conf. on Intelligent Systems, (2006), 716721

25. Yager,R.R,On the theory of bags,(Multi sets), International Journal of General System, 13(1986), 23 37.

26. Yanhong.L, Olson.D, Qin.Z., Similarity between Intuitionistic fuzzy sets: Comparative Analysis, Pattern Recognition Letters, 28(2) (2007), 278-285.

27. Ye.J, Cosine similarity measures for intuitionistic fuzzy sets and their applications, Mathematical and Computer Modelling, vol.53(2011), 91-97.

28. Zadeh.L.A., Fuzzy Sets, Information and Control, 8 (1965), 338-353. 